\title{
Research into Liyuan Buildings, the Spatial Composition of Liyuan Blocks and Liyuan Residents’ Lifestyles in Qingdao, China
}

\author{
Limin Shao ${ }^{1}$, Hiroyuki Kanekiyo ${ }^{2}$ \\ ${ }^{1}$ Department of Architecture \& Landscape, Shandong University of Art \& Design, Jinan, China \\ ${ }^{2}$ Graduate School of Engineering, Kyushu University, Fukuoka, Japan \\ Email: limin6782000@aliyun.com
}

Received June $15^{\text {th }}$, 2013; revised July 22 ${ }^{\text {nd }}, 2013$; accepted August $12^{\text {th }}, 2013$

\begin{abstract}
Copyright ( 2013 Limin Shao, Hiroyuki Kanekiyo. This is an open access article distributed under the Creative Commons Attribution License, which permits unrestricted use, distribution, and reproduction in any medium, provided the original work is properly cited.
\end{abstract}

\begin{abstract}
Qingdao lies in the south of Shandong Peninsula, on the coast of the Yellow Sea. As a coastal and hilly city, Qingdao has topographic features consisting of twisted coastlines dotted with capes and bays. The beginning of the 20th century witnessed the construction of western-style streets and buildings in Qingdao when it was occupied by Germany. Liyuan is the name of the residential buildings for Chinese labors during the German occupation. Liyuan retain the spatial composition of Chinese quadrangles (Siheyuan), and at the same time feature western technology. Therefore they have both Chinese and western flavors. In addition, they are the earliest urban residential complexes in China. The space system of Liyuan blocks has played an important role in the lifestyle and social network of the local residents. Liyuans have been home to Qingdao people from generation to generation, which makes it a spiritual symbol. Owing to its history and characteristics, Liyuan architecture has been designated as one of the historical and cultural protected areas in Qingdao, and has contributed to the formation of a distinctive geographical landscape. Since the 1980s, urban renewal and redevelopment in Qingdao have greatly impacted upon the protection of Liyuans, which has aroused concern from various communities. This research on Liyuans shows the geographic location of all the Liyuan blocks, classifies them in terms of courtyard composition and architectural features, and analyzes the relationship between their spatial composition and the residents' lifestyle.
\end{abstract}

Keywords: Qingdao City; Liyuans; Liyuan Block; Neighborhood; Social Networks

\section{Introduction}

Hundreds of years ago, the Liyuan-Qingdao people's dwelling was facing urban redevelopment such as functional renewal and demolition, which brought great impact on Qingdao's landscape features. As the Liyuan has been listed as one of Qingdao's historical and cultural conservation areas, the study of its formation, distribution, current situation and the daily life of the residents has become a common topic for people from all walks of life. Based on long-term investigation and research, this study shows the current distribution of the Liyuan, thus providing reference for further research.

\section{Study Purpose and Method}

This study has made use of topographical maps and aerial photos as well as referring to many research methods such as literature research, field research and inquiry. With the fundamental knowledge of the exterior and interior structure of its architecture, the study has come to understand the relationship between spatial structure and people's living behavior and also the conditions and process for the formation of social networks around the Liyuan blocks.

\section{Formation and Development of the Liyuan}

The Liyuan were built by Germans for the residence of Chinese people during its colonial domination of Qingdao from 1898 to 1914 . Their style resembles an elevated quadrangle courtyard or building yard which has combined the space structure of traditional Chinese courtyards with western architectural technology. Centering around the courtyard, the building yard is surrounded by two sides, three sides or four sides. At the very beginning, they were made of bricks and wood with two or three floors, followed later by four or five floored concrete structures. The first floor was used for storage or factories, while the upper floors were residential. This is the earliest congregated housing available for renting in modern cities.

Most of the Liyuan were built between 1920 and 1930. According to statistics provided by Qingdao's Social Affairs Bureau, in 1933 there were approximately 506 yards, 10,669 households and 16,701 rooms.

\section{Record of the Earliest Liyuan for Chinese Laborers}

“'The Illustarte Zeitung' published with photos on 9th, Feb. 1899 that residential housing for 500 Chinese labors employed 
by private companies had been built for the guarantee of abundant labor requirement" (Klaus, 2000).

"You can also see the orderly tartan blocks nearby the harbor which is located in the northern part of city planning at that time and along current Jimo road. They built houses for 500 Chinese labors at the harbor" (Klaus, 2000).

\section{Site Selection of the Liyuan and Their Relation to the Surrounding Environment}

According to the General Program of Management of East and West Towns on Bao Island in Qingdao, it marked out the European Residential Area, namely Qingdao District, which occupied the best area. The Residential Area for Chinese people was divided into Big Bao Island, Taidong and Taixi (Xinhua news Agency, 1999).

From their formation, the Liyuans were intended for manual laborers, therefore their design standards, be it in street width or building density, were far from being comparable with the German villas.

\section{Formation of Residential Hierarchy}

From its earliest building intent, site selection analysis and targeted users of Chinese workmen, it's obvious that they chose places near Chinese laborers and assignments as the criteria for the site selection.

In 1912 the partition ban was abolished and Chinese people began to live in the urban area of Qingdao with improved living conditions. Then the Liyuans gradually developed into the most common residential style. Though their hierarchy was changed to some extent, yet they still lived in lower-middle-class areas compared with other residences (Figure 1).

\section{Space Characteristics of Existing Liyuan and Its Utilization}

\section{Road System of Liyuan Blocks and Its Combination with Buildings and Courtyards}

1) Road System

In accordance with the width of blocks road, the blocks road fall into neighborhood road and inside-neighborhood alleys. We found during our survey that courtyards in the blocks are not directly connected to its road, but through the interconnected Inside-neighborhood alleys. The streets with a Liyuans are found in the following neighborhood areas in 2011: E11: Ningyang Road, F27: Jining sub-road. All of them have formed the street space.

Based on the distribution of Liyuan existing before May, 2011, this study has classified as follows for the benefits of research and analysis:

District A: to the north: Qingcheng Road, to the south: Huaitai Road, to the northwest: Qingcheng Road, to the east: Leling Road, 5 neighborhoods in all.

District B: to the north: Liaoning Road, to the south: Jilin sub-road, to the northwest: Liaoning Road, to the east: Shouguang Road

District C: to the north: Enxian Road, to the south: No. 1 Shichang Road and Xiajin Road, to the west: Laizhou Road, to the southeast: Deping Road, 20 neighborhoods in all.

District D: to the northeast: Dalian Road, to the southeast: Jiaozhou Road, to the northwest: Wudi Road, 5 neighborhoods in all.

District E: to the northwest: Jinan Road, to the south: Hubei Road, to the west: Taian Road and Jinan Road, to the east: Zhongshan Road, 17 neighborhoods in all.

District F: to the north: Cangkou Road, to the southeast: Qufu Road and Anhui Road, to the west: Zhongshan Road, to the northeast: Jining Road and Pingyuan Road, 32 neighborhoods in all.

District G: to the west: Sichuan Road, to the southeast: Chengwu Road, to the southwest: Cishan Road, to the east: Guancheng Road, 7 neighborhoods in all (Figure 2).

2) Courtyard Combination

a) Separate Courtyard: (F16) Guang Xingli, as the separate courtyard in the whole neighborhood, is regarded from recorded history as the Liyuans with the most households and the largest yard space, especially when there are fewer separate Liyuan present today.

b) Multiple Courtyards: There is separate courtyard and multiple courtyards based on the architectural enclosed relationship. All of them include entrances which lead to block road and form the frequently seen yards.

c) Interconnected Courtyard: a) Along the street (A1), Qingcheng Road, (D5) No. 3 Wudi Road, b) interconnected from right and left: (E5) No. 5 Shanxi Road (Figure 3).

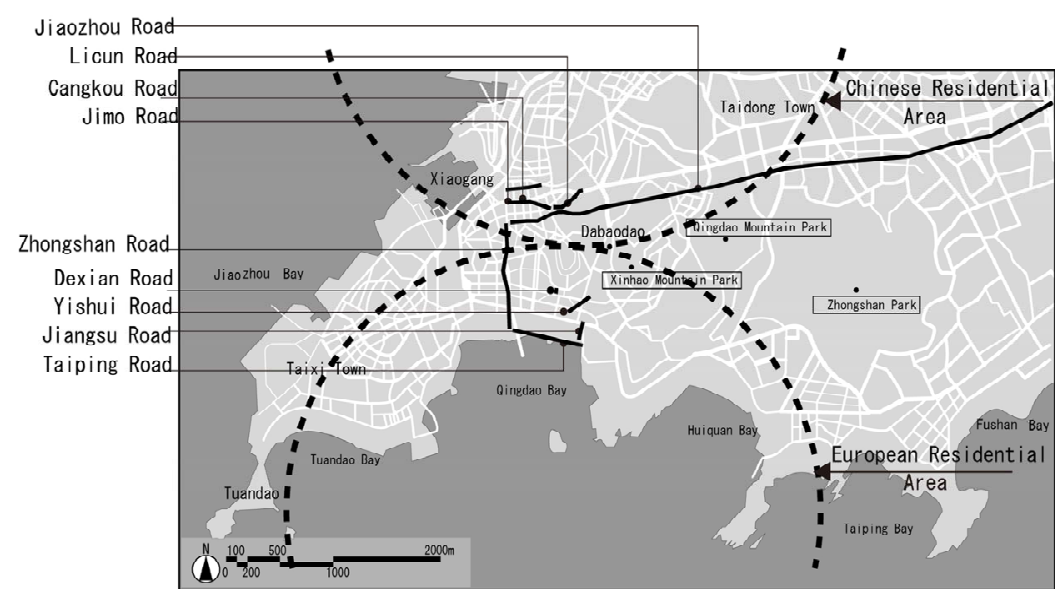

Figure 1.

Map of Chinese and European residential area. 


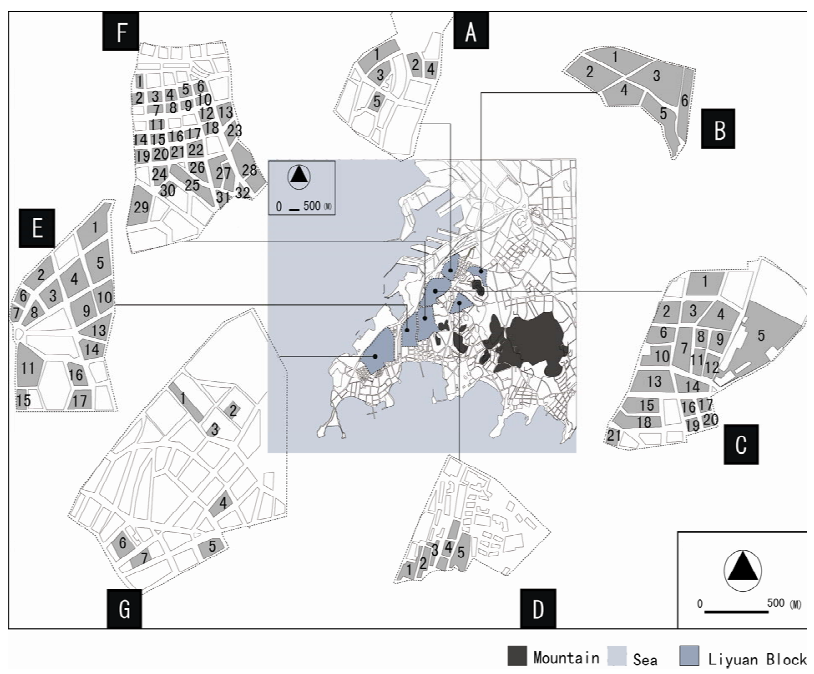

Figure 2.

Distribution of the existing inner yards in Qingdao.

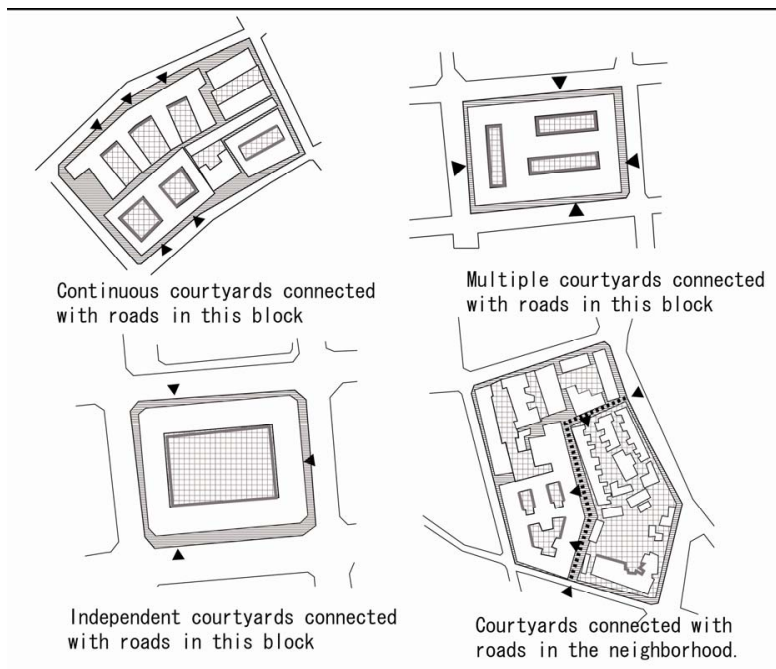

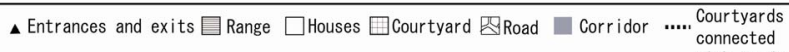

Figure 3.

Road system of Liyuan blocks and its combination with buildings and courtyards.

\section{Spatial Form and Categories of the Liyuan}

Situated mostly on hilly lands and areas of fluctuating terrain, the Liyuan adopted partial sinking in vertical set and have been arranged into different units by bordering along the contour line, thus developing into variable combinations and architectural forms according to topographic altitude.

1) Street Corner

The city planning during the period of German domination paid great attention to the diagonal of streets; therefore, features of architectures on the corner of the neighborhoods have been carefully handled and highlighted. It has always been regarded as the symbol of the block's image and presented at the high building, entrance door in a facade manner.

Besides, Liyuan at the corner always hold special spatial effect and visual focus.
2) Ornament of inside and outside Façade

Features of the Liyuan are: a) storied house; b) enclosed courtyard; c) combination of Chinese and western elements in ornamentation.

The Liyuan is viewed as a combination of western architectural style and Chinese quadrangle courtyard. Buildings facing the streets have adopted a western style which is manifest in walls, roofs and patterns and decoration on the entrance doors. However, the interior decoration, which is totally different from the exterior decoration, has used Chinese colored drawing, wooden plaid and screen walls.

3) Coexistence of Old and New Liyuans

The field survey conducted from 2009 to 2011 indicated that some of the preserved Liyuans were built after the foundation of People's Republic of China or the Reform and Opening-up. Though they're brick-concrete houses, their combination form conform fully to the Liyuan style and constitute the coexistence of old and new yards.

4) Interior Ornament

Its exterior façade obviously displays western architectural style, while the interior elements and decoration manifest Chinese style. Basically, it's made of bricks and woods. Though wood is the main material, its wall base, like its western counterparts, uses stones. As with Chinese traditional dwellings, there is a screen wall at the entrance door. The Liyuan is generally of veranda style and boasts lots of carved beams and painted rafters existing today.

5) Beautifying

People in the Liyuan like planting flowers and growing bonsais. They always plant fig trees, which seem to be symbolic. Other plants include metasequoia and loquat. The well-preserved decorations on roof, under the eaves, in corridors and between columns, are able to show the highlights of Liyuan in the past days (Figure 4).

\section{Functional Allocation of Space System of Liyuans}

Based on the spatial features of Liyuan, this study comprises interior spatial system and exterior spatial system. The interior elements contain indoors, corridors, stairs, inside yards and entering-and-exiting buildings. The exterior space includes streets, surrounding areas, street intersections, the entrance and exit of Liyuan and hilltop parks.

1) Interior Space:

a) Indoor Space

According to investigation by Guang Xingli, there are slight differences in space combinations between the west, north, east and south buildings. The depth of the west building is about 6.2 $\mathrm{m}$ and its front width is about $3.8 \mathrm{~m}$, while the depth of the north, east and south buildings is between 5.4 and $5.5 \mathrm{~m}$ and the front width is $2.6-2.8 \mathrm{~m}$.

b) Corridor Space

Considering the space limit, the width of the corridor is designed as $1.2 \mathrm{~m}$, which is not only the route for connecting each household, but also a place for drying clothes, cooking and washing. Furthermore, stoves, honeycomb briquette and other stuff are also arranged here.

c) Inside Yards Space

In the past, yards in Guang Xingli had temples and public wells. Many old people dried their clothes, read newspapers and dozed off in those places near their houses. People gathered 

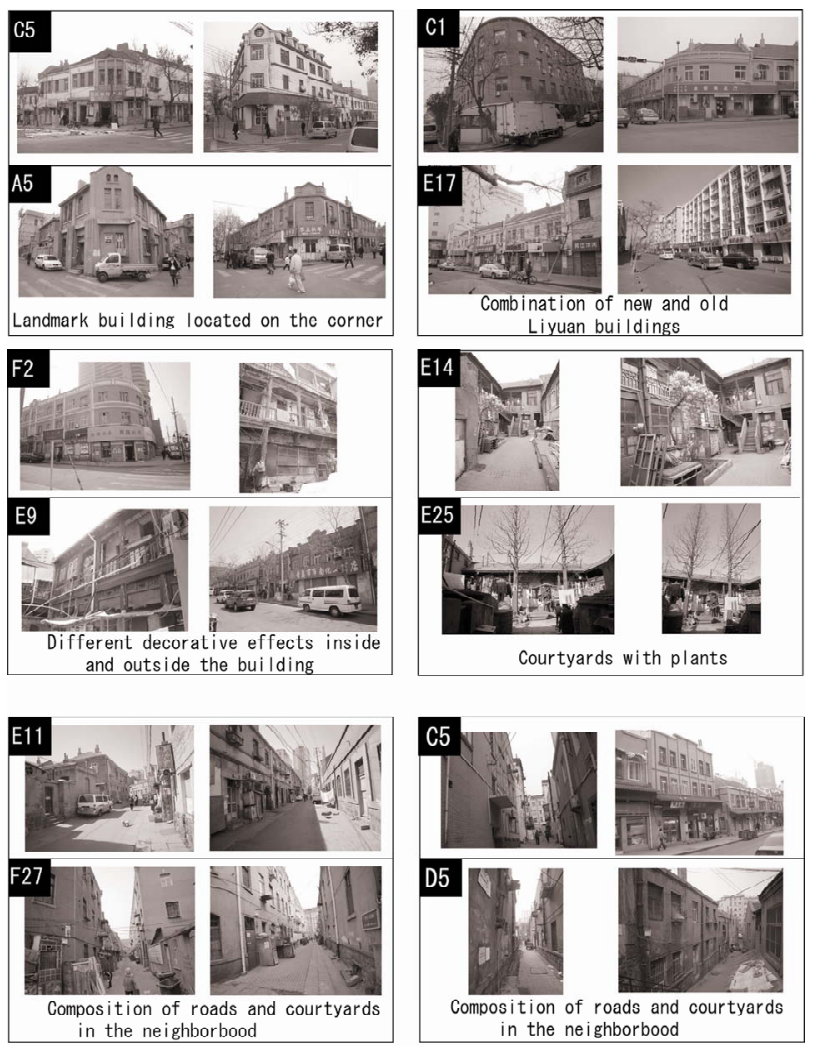

Figure 4.

Liyuan which are well maintained.

here to chat, play cards, drink alcohol, dry clothes, do housework, play with children, relax in the coolness and sometimes simply to kill time. These places became gathering places to spend their leisure time. It's recorded that people also watched movies and performed in operas here and this place was once used as a small factory.

2) Exterior Space System

It refers to the space outside buildings, namely, the space of streets, shop arcades, street intersections and hilltop parks.

a) Street Space, Shops and Factories

Many western-style shopping, entertainment and living buildings were established along southern Zhongshan Road in the German period. The northern area, which was lived in by Chinese people, was full of stores and vendors. Silk cloth shops and traditional famous snack bars run by national capitalists were scattered here and there, such as "Qian Xiangyi”, "Rui Fuxiang”, "Chun Helou”.

Zhongshan Road was a prosperous area in the 1920s with intensive Liyuan located around its surrounding streets. Those streets formed by enclosed Liyuans were also places for purchasing goods for the spring festival (Shao \& Kanekiyo, 2008).

b) Shops

Axe Fire wood Court (Pichaiyuan) on the Zhongshan road is a gathering place for many folk cultures, including wharf culture, urban culture, business culture, cuisine culture and opera culture (Shao \& Kanekiyo, 2009).

\section{Establishment of Urban Social Networking in Space System of Liyuan}

\section{1) Hierarchical Social Networking}

The urban sociology indicates that people in the Liyuan share common interests and values in life. Valuing family and neighborhood relationships, they are sincere and friendly to their friends and neighbours but also exclusive to others. It constitutes a great contrast with modern residents who share no common ideology and care nothing about each other.

2) Formation of Life Networking

The formation of life networking depends on a specific physical environment and living conditions. The survey shows that areas around stores are both shopping places and places of intercourse. Street intersections and entrances and exits of Liyuans are always used as activity place and intercourse place. The favorable space for communication created by their common life background and yard pattern has helped them to develop their working, living and communication networking.

\section{Conclusion}

Since 2008 more than 4 years of field investigations have been conducted for this survey. This study, with comprehensive investigations on the distribution and status quo of Liyuan before March, 2010, has also categorized them according to their architectural and spatial characteristics and offered complete materials for further studies in this field.

During years of study we have vividly felt the neighborhood relationships of harmony, tolerance, mutual help and cooperation, and the concept of mutual support and common action, in the way it has developed the urban social networking in inner yard architectural system and hierarchic urban space.

The space structure and existing social living system have developed favorable results in aspects such as community environment and social communication. The survey has noticed that new residence buildings have been set up among old Liyuan buildings, which has greatly improved plot ratio and extended social networking under the basis that modern requirements have been satisfied.

Therefore, it can be concluded that we need to preserve these small-scale social networking structures rather than simply update, renew and change functional uses during the Liyuan's renovation. The hierarchic space structure and complete social networking development of Liyuan can still offer beneficial reference to city planners in modern times.

\section{REFERENCES}

Klaus, M. (2000). The domination and resistance of the model colony Kiaochou Bay, interaction between China and Germany in 18971914. München: R. Oldenbourg Verlag.

(1999). Qingdao city planning, annals of Qingdao (pp. 28-30). Beijing: Xinhua News Agency.

Shao, L. M., \& Kanekiyo, H. (2008). Investigation on city landscape and the use of public space in Fukuoka in Japan and Qingdao in China. Japanese Gardening Association, Jiuzhou Branch, 16, 11-12.

Shao L. M., \& Kanekiyo, H. (2009). Liyuan, Liyuan districts conservation investigation. Japanese Gardening Association, Jiuzhou Branch, 17, 9-10. 